\title{
Carduus tenuiflorus Curtis (Asteraceae), nuevo registro para la flora alóctona de Chile
}

\section{Carduus tenuiflorus Curtis (Asteraceae), new record for the alien flora of Chile}

\author{
Sebastián Cordero ${ }^{1 *}$, Diego G. Gutiérrez $Z^{2}$ \& Andrés Moreira-Muñoz ${ }^{3}$ \\ ${ }^{1}$ Instituto de Biología, Facultad de Ciencias, Pontificia Universidad Católica de Valparaíso, Campus Curauma, Avenida \\ Universidad 330, Valparaíso, Chile. \\ ${ }^{2}$ División Plantas Vasculares, Museo Argentino de Ciencias Naturales, MACN-CONICET, Avenida Ángel Gallardo 470, \\ C1405DJR, Buenos Aires, Argentina. \\ ${ }^{3}$ Instituto de Geografía, Facultad de Ciencias del Mar y Geografía, Pontificia Universidad Católica de Valparaíso, Avenida \\ Brasil 2241, Valparaíso, Chile. \\ *sebastian.cordero.r@mail.pucv.cl
}

\begin{abstract}
Carduus tenuiflorus Curtis is described as a new alien plant species for Chile, whose introduction into the country is proposed around the year 1928. An identification key for species of the genus Carduus L. present in Chile is added.
\end{abstract}

El género Carduus L. pertenece a la familia con mayor riqueza de plantas vasculares de Chile (Zuloaga et al. 2008, Moreira-Muñoz et al. 2012) y el mundo, Asteraceae, con unos 1.033 géneros y 24.000-30.000 especies (Funk et al. 2009). Este género comprende aproximadamente 90 especies nativas de Eurasia, y norte y este de África, muchas de las cuales han sido introducidas en América y Australia (Bremer 1994, Kadereit \& Jeffrey 2007). En Chile han sido citadas tres especies alóctonas: Carduus nutans L., Carduus pycnocephalus L. y Carduus thoermeri Weinm. (Matthei 1995, Zuloaga et al. 2008, Fuentes et al. 2013, Ray et al. 2014).

Carduus tenuiflorus Curtis es una especie originaria de Europa y norte de África e introducida en distintas partes del mundo (Parsons \& Cuthbertson 1992), de hábitat ruderal, preferentemente nitrófila y de gran similitud con $C$. pycnocephalus, con la cual es confundida ocasionalmente (Devesa \& Talavera 1981). Aunque C. tenuiflorus y C. pycnocephalus son especies bastante próximas, se consideran independientes y válidas (Kazmi 1964), sin embargo, su determinación resulta dificultosa debido su afinidad morfológica y la superposición de sus áreas de distribución.

En 2015, en el marco de estudios florísticos realizados en diversas localidades de la región de Valparaíso, se detectaron dos poblaciones de C. tenuiflorus, una especie no registrada para Chile. Por otro lado, se examinaron especímenes depositados en los herbarios CONC, SGO y UCVA, con el fin de determinar la posible fecha de introducción de la especie en Chile.
DESCRIPCIÓN DE LA ESPECIE

Carduus tenuiflorus Curtis, Fl. Londin. 6(61): tab. 55. 1789.

Sinónimos: Carduus pycnocephalus L. var. tenuiflorus (Curtis) Ball, J. Linn. Soc., Bot. 16: 521. 1878. Carduus pycnocephalus L. var. tenuiflorus (Curtis) Fiori \& Bég., comb. superfl., Fl. Ital. 3: 360. 1904.

Planta anual o bienal de hasta $150 \mathrm{~cm}$ de altura. Tallos erectos, simples o ramificados en la mitad superior, con alas dentado-espinosas más o menos anchas y no interrumpidas en toda su longitud. Hojas sésiles, láminas pinnatífidas o pinnatipartidas, las inferiores con 4-6 pares de lóbulos palmeado-espinosos, base decurrente, margen con indumento lanoso, laxo en el haz y más denso en el envés. Capítulos sésiles o subsésiles, en grupos de (2-)3-10(-12) en el extremo de las ramitas. Involucro de $15-18 \mathrm{~mm}$ de alto, cilíndrico-acampanado; filarios oblongo-lanceolados, acuminados, los medianos e internos con margen escarioso, glabros o algo lanuginosos en el dorso. Flores con corola tubulosa, purpúrea, 5-secta, con tubo de 4-6 $\mathrm{mm}$ de largo y limbo de 6-9 mm. Aquenios elipsoides, de 3,5-4,5 $\mathrm{mm}$ de largo, levemente comprimidos, glabros, lustrosos, con rayas obscuras longitudinales. Papus caedizo, de $12-15 \mathrm{~mm}$ de largo, con pelos escábridos, soldados en la base (Fig. 1).

HÁBITAT Y ECOLOGÍA

En la región de Valparaíso, C. tenuiflorus fue registrado en dos localidades: Parque Natural Gómez Carreño, ubicado al costado norte de la ciudad de Viña del Mar y cerros colindantes al sector de Los Pinos, Quilpué. 
Las poblaciones de esta especie habitan laderas asoleadas, con suelos fuertemente erosionados y se encuentran generalmente asociadas a otras herbáceas alóctonas tales como Carthamus lanatus L., Hordeum murinum L., Lolium perenne L., Papaver somniferum L. y Silybum marianum (L.) Gaertn. También crecen entre gramíneas y vegetación baja formada principalmente por renovales de Acacia caven (Molina) Molina y Senna candolleana (Vogel) H.S. Irwin \& Barneby, formando grupos monoespecíficos de 15 a 30 individuos que viven simpátricamente con C. pycnocephalus.
Otras poblaciones se desarrollan en áreas urbanas altamente perturbadas a causa de la actividad antrópica, colonizadas exclusivamente por especies alóctonas, dentro de las que destacan Avena barbata Pott ex Link, Chrysanthemum coronarium L., Genista monspessulana (L.) L.A.S. Johnson y Malva nicaeensis All.

C. tenuiflorus presenta crecimiento vegetativo desde el mes de septiembre, su floración se produce en octubre y su senescencia en diciembre.
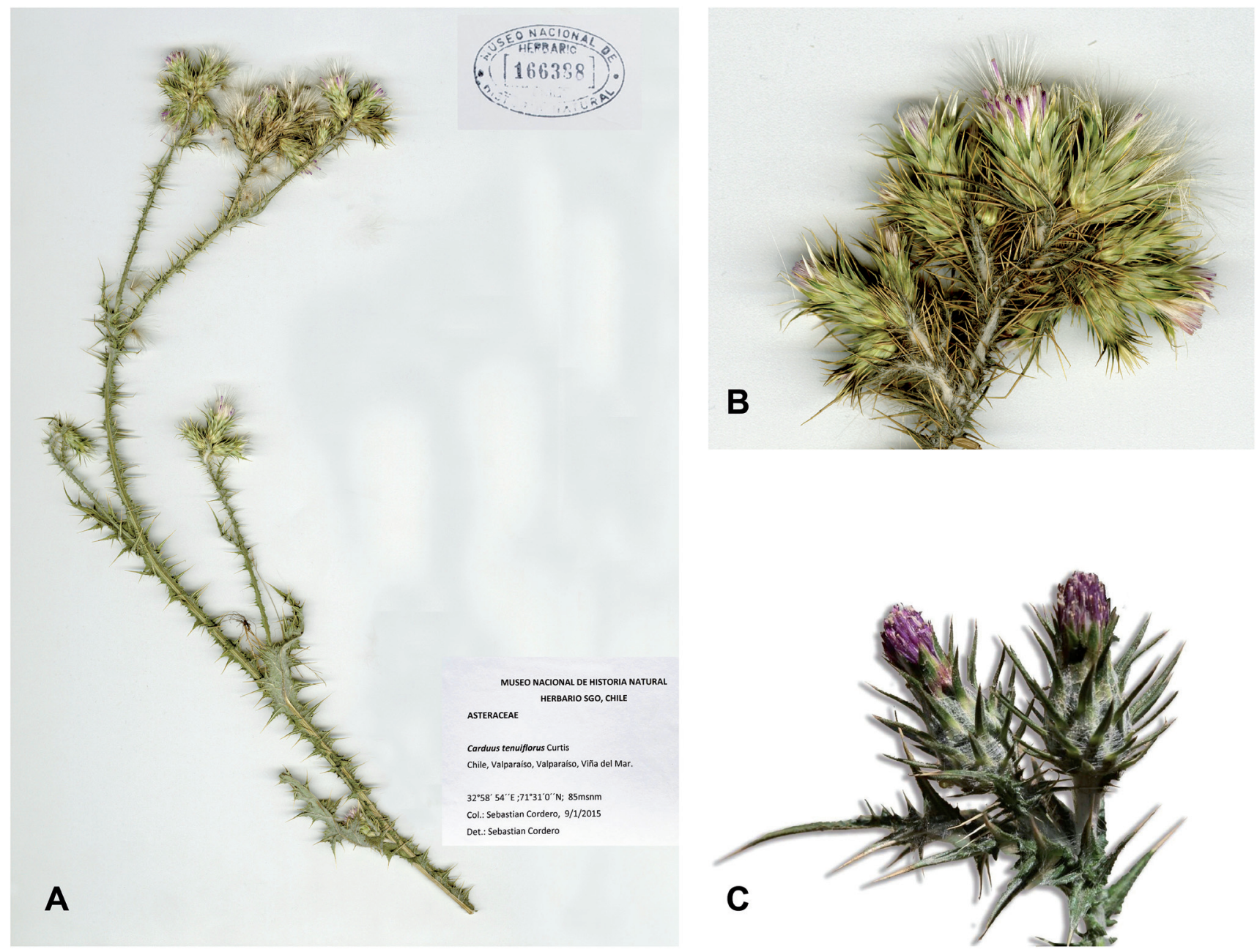

Figura 1. A. Carduus tenuiflorus, Cordero s.n (SGO 166389); B. Carduus tenuiflorus, detalle de capítulos; C. Carduus pycnocephalus, detalle de capítulos (Fotografía de S. Cordero).

Figure 1. A. Carduus tenuiflorus, Cordero s.n (SGO 166389); B. Carduus tenuiflorus, capitula detail; C. Carduus pycnocephalus, capitulua detail (Photograph by S. Cordero). 
Material adicional ESTUdiado

CHILE, Región de Coquimbo, Prov. Choapa, Los Vilos. 3-XI-2006. Tepe et al. 1702 (CONC); Región de Valparaíso, Prov. Marga Marga, Quilpué. 18-XI-1928. Garaventa 316 (CONC); Quilpué, sector de Los Pinos, 3304'39' S; 71²5'34'”. 166 m, X-2015, Cordero s.n. (SGO 166388); Prov. Valparaíso, Parque Natural Gómez Carreño, 32 58'54"S 71³1'00"'W, 85 m, IX-2015. Cordero s.n. (SGO 166389); Isla Robinson Crusoe. 21-VIII-2002. Cuevas s.n. (CONC 158046); Región del Maule, Prov. Talca, Constitución. 3-XI-1958. Barnier 271 (CONC); Región de Biobío, Prov. Concepción, Arenales de San Vicente. 27-XI1934. Junge s.n. (CONC 5139); Isla Rocuant. 9-XI-2000. Baeza \& López 101 (CONC).

\section{Comentarios}

Se revisaron pliegos determinados como C. pycnocephalus de los herbarios CONC, SGO y UCVA con el fin de encontrar entre ellos especímenes correspondientes a $C$. tenuiflorus pues, debido a su gran similitud morfológica, éstos podrían haberse considerado como una sola entidad. En CONC se detectaron seis especímenes correspondientes a C. tenuiflorus, mientras que en SGO y UCVA ninguno. El espécimen más antiguo encontrado fue recolectado en el año 1928 (CONC 48897), lo cual permite suponer que la introducción de la especie en Chile tuvo lugar hace casi un siglo. Cabe destacar que el año de introducción de C. pycnocephalus en Chile fue propuesto en base a un espécimen actualmente determinado como C. tenuiflorus (CONC 48897), por lo cual es necesario resolver la posible fecha de introducción de esta especie en el país. El rango de distribución de C. tenuiflorus en Chile, sobre la base de los datos existentes hasta el momento, se define desde la IV Región de Coquimbo a la VIII Región de Biobío, incluyendo el Archipiélago Juan Fernández (Fig. 2).

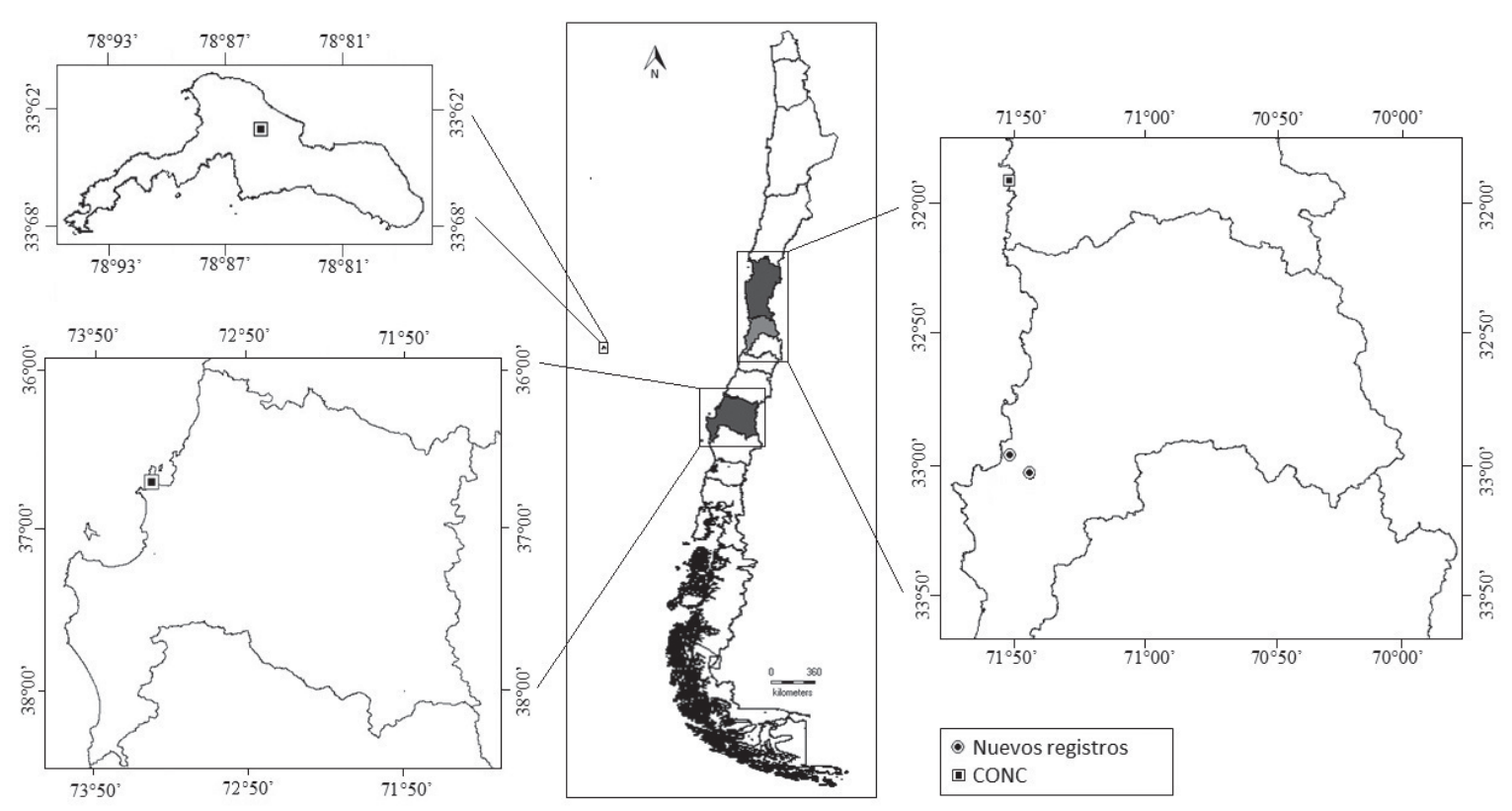

Figura 2. Localización de los especímenes estudiados de C. tenuiflorus.

FiguRE 2. Location of studied specimens of C. tenuiflorus.

\section{Clave para las especies de Carduus en Chile}

1. Capítulos pequeños, en grupos; involucro cilíndrico-ovoideo o cilíndrico-acampanado; plantas lanuginosas.

2. Filarios más o menos lanosos, no escariosos en el margen; involucro de 6-12 mm de alto y 8-10 mm de

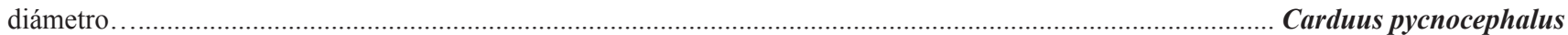
2'. Filarios glabros o casi glabros, escariosos en el margen; involucro de $15-18 \mathrm{~mm}$ de alto y $10-14 \mathrm{~mm}$ de diámetro.

1'. Capítulos muy grandes, solitarios; involucro ovoide, globoso o hemisférico; plantas glabras o casi glabras.

3. Filarios de 1,5-5 mm de ancho, glabros o muy levemente pubérulos; capítulos con pedúnculos hasta de $15 \mathrm{~cm}$ Carduus tenuiflorus

3'. Filarios de 4-8 $\mathrm{mm}$ de ancho, conspicuamente pubérulos; capítulos con pedúnculos hasta de $22 \mathrm{~cm}$

Carduus nutans Carduus thoermeri 
Las especies $C$. nutans y $C$. thoermeri han sido citadas para Chile (e.g. Matthei 1995, Zuloaga et al. 2008, Fuentes et al. 2013). En este trabajo se siguen los criterios taxonómicos de Kazmi (1964), Franco (1976) y Devesa \& Talavera (1981), en los que se consideran a ambas especies como independientes. Sin embargo, dada la taxonomía compleja de estas especies introducidas en América del Sur, estudios futuros de sus poblaciones a ambos lados de la Cordillera de los Andes serán necesarios para determinar la real identidad de estos dos taxones en nuestro continente.

\section{AGRADECIMIENTOS}

A Fondecyt 1150425 por financiar parte de este trabajo. Se agradece a los curadores de SGO (Gloria Rojas) y CONC (Alicia Marticorena), el acceso al material de consulta.

\section{BIBLIOGRAFÍA}

Bremer, K. 1994. Asteraceae. Cladistics and Classification. Timber Press, Portland. 752 pp.

Devesa, J.A. \& S. Talavera. 1981. Revision del género Carduus (Compositae) en la Península Ibérica e Islas Baleares. Universidad de Sevilla, España. 118 pp.

Franco, J.D.A. 1976. Carduus. In: T.G. Tutin, V.H. Heywood, N.A. Burges, D.M. Moore, D.H. Valentine, S.M. Walters \& D.A. Webb (eds.), Flora Europaea, Vol. 4, pp. 220-232. Cambridge University Press, Cambridge, UK.
Fuentes, N., A. Pauchard, P. SÁnchez, J. Esquivel \& A. Marticorena. 2013. A new comprehensive database of alien plant species in Chile based on herbarium records. Biological Invasions 15(4): 847-858.

Funk, V.A., A. Susanna, T.F. Stuessy \& R.J. Bayer (eds.). 2009. Systematics, Evolution, and Biogeography of Compositae. International Association for Plant Taxonomy. Institute of Botany, University of Vienna. 965 pp.

Kadereit, J.W. \& C. Jeffrey. 2007. Flowering plants. Eudicots: Asterales. In: K. Kubitzki (ed.), The families and genera of vascular plants, Vol. 8, pp. 61-588. Springer, Berlín, Gemany.

Kazmi, S.M.A. 1964. Revision der Gattung Carduus (Compositae). Teil II. Mitteilungen der Botanischen Staatssammlung München 5: 279-550.

Matthei, O. 1995. Manual de las Malezas que crecen en Chile. Alfabeta Impresiones, Santiago de Chile. 545 pp.

Moreira-Muñoz, A., V. Morales \& M. Muñoz-Schick. 2012. Actualización sistemática y distribución geográfica de Mutisioideae (Asteraceae) de Chile. Gayana Botánica 69: 9-29.

Parsons, W.T. \& E. G. Cuthbertson. 1992. Noxious Weeds of Australia. Inkata Press, Melbourne. 692 pp.

Ray, C.A., V. Escobedo-Echeverría, S.A. Castro \& F.M. Jaksic. 2014. Catálogo de la flora y fauna invasora en Chile continental e insular oceánico. En: F. Jaksic \& S. Castro (eds.), Invasiones Biológicas en Chile: Causas globales e impactos locales, pp. 491-505. Ediciones UC, Santiago de Chile.

Zuloaga, F.O., O. Morrone \& M.J. Belgrano (eds.). 2008. Catálogo de las plantas vasculares del Cono Sur (Argentina, Sur de Brasil, Chile, Paraguay y Uruguay), Vol. 2. pp. 9852286. Missouri Botanical Garden Press, St. Louis.

Recibido: 25.04.16

Aceptado: 04.07.16 